\title{
Some isomorphically polyhedral \\ Orlicz sequence spaces
}

\author{
DENNY H. LEUNG
}

\begin{abstract}
A Banach space is polyhedral if the unit ball of each of its finite dimensional subspaces is a polyhedron. It is known that a polyhedral Banach space has a separable dual and is $c_{0}$-saturated, i.e., each closed infinite dimensional subspace contains an isomorph of $c_{0}$. In this paper, we show that the Orlicz sequence space $h_{M}$ is isomorphic to a polyhedral Banach space if $\lim _{t \rightarrow 0} M(K t) / M(t)=\infty$ for some $K<\infty$. We also construct an Orlicz sequence space $h_{M}$ which is $c_{0^{-}}$ saturated, but which is not isomorphic to any polyhedral Banach space. This shows that being $c_{0}$-saturated and having a separable dual are not sufficient for a Banach space to be isomorphic to a polyhedral Banach space.
\end{abstract}

A Banach space is said to be polyhedral if the unit ball of each of its finite dimensional subspaces is a polyhedron. It is isomorphically polyhedral if it is isomorphic to a polyhedral Banach space. Fundamental results concerning polyhedral Banach spaces were obtained by Fonf [1, 2].

Theorem 1 (Fonf) A separable isomorphically polyhedral Banach space is $c_{0}$-saturated and has a separable dual.

Recall that a Banach space is $c_{0}$-saturated if every closed infinite dimensional subspace contains an isomorph of $c_{0}$. Fonf also proved a characterization of isomorphically polyhedral spaces in terms of certain norming subsets in the dual. In order to state the relevant results, we introduce some terminology due to Rosenthal [4, 5]. The (closed) unit ball of a Banach space $E$ is denoted by $U_{E}$.

Definition Let $E$ be a Banach space.

(1) A subset $W \subseteq E^{\prime}$ is precisely norming (p.n.) if $W \subseteq U_{E^{\prime}}$, and for all $x \in E$, there is a $w \in W$ such that $\|x\|=|w(x)|$.

(2) A subset $W \subseteq E^{\prime}$ is isomorphically precisely norming (i.p.n.) if $W$ is bounded and

1991 Mathematics Subject Classification 46B03, 46B20, 46B45. 
(a) there exists $K<\infty$ such that $\|x\| \leq K \sup _{w \in W}|w(x)|$ for all $x \in E$,

(b) the supremum $\sup _{w \in W}|w(x)|$ is attained at some $w_{0} \in W$ for all $x \in E$.

It is easy to see that $W \subseteq E^{\prime}$ is i.p.n. if and only if there is an equivalent norm \|\|$\cdot \|$ on $E$ so that $W$ is p.n. in $(E,\|\| \cdot \| \mid)^{\prime}$.

Theorem 2 (Fonf) Let $E$ be a separable Banach space. Then $E$ is isomorphically polyhedral if and only if $E^{\prime}$ contains a countable i.p.n. subset.

This paper is devoted mainly to the problem of identifying the isomorphically polyhedral Orlicz sequence spaces. In $\S 1$, we prove a characterization theorem for isomorphically polyhedral Banach spaces having a shrinking basis. This result is applied in $\S 2$ to obtain examples of isomorphically polyhedral Orlicz spaces. In $\S 3$, a nonisomorphically polyhedral, $c_{0}$-saturated Orlicz sequence space is constructed. Since every $c_{0}$-saturated Orlicz sequence space has a separable dual, this shows that the converse of Theorem 1 fails, answering a question posed by Rosenthal [4.

Standard Banach space terminology, as may be found in [3], is employed. If $\left(e_{n}\right)$ is a basis of a Banach space $E$, and \|\|$\cdot \mid \|$ is a norm on $E$ equivalent to the given norm, we say that $\left(e_{n}\right)$ is monotone with respect to $\||\cdot|\|$ if $\left\|\left|\sum_{n=1}^{k} a_{n} e_{n}\right|\right\| \leq\left\||| \sum_{n=1}^{k+1} a_{n} e_{n}\right\| \|$ for every real sequence $\left(a_{n}\right)$ and all $k \in \mathbb{N}$. Terms and notation regarding Orlicz spaces are discussed in $\S 2$.

\section{A characterization theorem}

This section is devoted to proving the following characterization theorem. Readers familiar with the proofs of Fonf's Theorems will find the same ingredients used here.

Theorem 3 Let $\left(e_{n}\right)$ be a shrinking basis of a Banach space $(E,\|\cdot\|)$. The following are equivalent.

(a) E is isomorphically polyhedral;

(b) There exists an equivalent norm $\||\cdot|\|$ on $E$ such that $\left(e_{n}\right)$ is a monotone basis with respect to $\|\cdot\| \cdot \|$, and for all $\sum a_{n} e_{n} \in E$, there exists $m \in \mathbb{N}$ such that

$$
\left\|\left|\sum _ { n = 1 } ^ { \infty } a _ { n } e _ { n } \left\|\left|=\left\|\mid \sum_{n=1}^{m} a_{n} e_{n}\right\| .\right.\right.\right.\right.
$$


Proof: Let $\left(P_{n}\right)$ be the projections on $E$ associated with the basis $\left(e_{n}\right)$. The sequence $\left(P_{n}\right)$ is uniformly bounded with respect to any equivalent norm on $E$. Also, $\left(P_{n}\right)$ converges strongly to the identity operator on $E$, which we denote by 1 . Since $\left(e_{n}\right)$ is shrinking, $\left(P_{n}^{\prime}\right)$ converges to $1^{\prime}$ strongly as well.

$(\mathrm{a}) \Rightarrow(\mathrm{b})$. By renorming, and using Theorem 2, we may assume that $E^{\prime}$ contains a p.n. sequence $\left(w_{k}\right)$. Fix sequences $\left(\epsilon_{k}\right)$ and $\left(\delta_{k}\right)$ in $(0,1)$ which are both convergent to 0 , and so that $\left(1+\epsilon_{k}\right)\left(1-2 \delta_{k}\right)>1$ for all $k$. For each $k$, choose $n_{k}$ such that $\left\|\left(1-P_{n}\right)^{\prime} w_{k}\right\| \leq \delta_{k}$ for all $n \geq n_{k}$. Define a seminorm $\|\cdot \mid\|$ on $E$ by

$$
\left\||| x\left|\|=\sup _{k}\left(1+\epsilon_{k}\right) \max _{1 \leq n \leq n_{k}}\right|\left\langle P_{n} x, w_{k}\right\rangle \mid .\right.
$$

Since $\left(w_{k}\right) \subseteq U_{E^{\prime}},\|\mid x\|\|\leq 2\| x\|\sup \| P_{n} \|$. On the other hand, if $x \neq 0$, choose $k$ such that $\|x\|=\left|w_{k}(x)\right|$. Then

$$
\begin{aligned}
\|x\| & =\left|w_{k}(x)\right| \leq\left|\left\langle x, P_{n_{k}}^{\prime} w_{k}\right\rangle\right|+\left|\left\langle x,\left(1-P_{n_{k}}\right)^{\prime} w_{k}\right\rangle\right| \\
& \leq\left|\left\langle P_{n_{k}} x, w_{k}\right\rangle\right|+\delta_{k}\|x\| .
\end{aligned}
$$

Thus

$$
\|x\|\left\|\geq\left(1+\epsilon_{k}\right)\left(1-\delta_{k}\right)\right\| x\|>\| x \| .
$$

Hence $\||\cdot| \mid$ is an equivalent norm on $E$. It is clear that $\left(e_{n}\right)$ is monotone with respect to \|\|$\cdot\|\|$. We claim that this norm satisfies the remaining condition in (b). To this end, we first show that the supremum in the definition (1) is attained. This is trivial if $x=0$. Fix $0 \neq x \in E$. Choose $k_{1} \leq k_{2} \leq \cdots$ and $\left(j_{i}\right), 1 \leq j_{i} \leq n_{k_{i}}$ for all $i$, so that

$$
\||x|\|=\lim _{i}\left(1+\epsilon_{k_{i}}\right)\left|\left\langle P_{j_{i}} x, w_{k_{i}}\right\rangle\right|
$$

We divide the proof into cases.

Case $1 \quad \lim _{i} k_{i}=\lim _{i} j_{i}=\infty$.

In this case, $P_{j_{i}} x \rightarrow x$ in norm. Therefore

$$
\limsup _{i}\left|\left\langle P_{j_{i}} x, w_{k_{i}}\right\rangle\right|=\limsup _{i}\left|\left\langle x, w_{k_{i}}\right\rangle\right| \leq\|x\| .
$$

Also, $\epsilon_{k_{i}} \rightarrow 0$ as $i \rightarrow \infty$. Thus, $\|\mid x\|\|\leq\| x \|$, contrary to (2).

$\underline{\text { Case } 2} \lim _{i} k_{i}=\infty, \lim _{i} j_{i} \neq \infty$.

By using a subsequence, we may assume that $j_{i}=j$ for all $i$. Then

$$
\left\|| | x \left|\left\|=\lim _{i}\left(1+\epsilon_{k_{i}}\right)\left|\left\langle P_{j} x, w_{k_{i}}\right\rangle\right| \leq\right\| P_{j} x \| .\right.\right.
$$


Now choose $k$ such that $\left\|P_{j} x\right\|=\left|\left\langle P_{j} x, w_{k}\right\rangle\right|$. If $j \leq n_{k}$,

$$
\begin{aligned}
\||x|\| & \geq\left(1+\epsilon_{k}\right)\left|\left\langle P_{j} x, w_{k}\right\rangle\right| \\
& =\left(1+\epsilon_{k}\right)\left\|P_{j} x\right\|>\left\|P_{j} x\right\|,
\end{aligned}
$$

a contradiction. Now assume $j>n_{k}$, then

$$
\begin{aligned}
\left\|\left(P_{j}-P_{n_{k}}\right)^{\prime} w_{k}\right\| & \leq\left\|\left(1-P_{j}\right)^{\prime} w_{k}\right\|+\left\|\left(1-P_{n_{k}}\right)^{\prime} w_{k}\right\| \\
& \leq 2 \delta_{k} .
\end{aligned}
$$

Hence

$$
\begin{aligned}
\left\|P_{j} x\right\| & =\left|\left\langle P_{j} x, w_{k}\right\rangle\right| \\
& \leq\left|\left\langle P_{n_{k}} x, w_{k}\right\rangle\right|+2 \delta_{k}\|x\| \\
& \leq\left(1+\epsilon_{k}\right)^{-1}|\|x\||\left|+2 \delta_{k}\right|\|x\| \| .
\end{aligned}
$$

Therefore,

$$
\left\||| x||\left|\leq\left\|P_{j} x\right\| \leq\left(\left(1+\epsilon_{k}\right)^{-1}+2 \delta_{k}\right)\right|\right\| x\||<\||| x \mid\|,
$$

reaching yet another contradiction. Consequently, we must have $\underline{\text { Case } 3} \quad \lim _{i} k_{i} \neq \infty$.

By using a subsequence, we may assume that the sequence $\left(k_{i}\right)$ is constant. Then it is

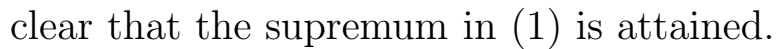

Now for any $x \in E$, choose $k$ so that the supremum in (1) is attained at $k$. Then it is clear that $\||x|\|=\left\|\mid P_{n_{k}} x\right\| \|$.

(b) $\Rightarrow(\mathrm{a})$. Let $\left(\eta_{n}\right)$ and $\left(\epsilon_{n}\right)$ be sequences convergent to 0 , with $1>\eta_{n}>\epsilon_{n}>0$ for all $n$. For each $n$, there is a finite $W_{n} \subseteq U_{(E,|\|\cdot\||)^{\prime}}$ such that

$$
\left(1+\epsilon_{n}\right)^{-1}\left|\left\|x \left|\left\|\leq \max _{w \in W_{n}}|w(x)| \leq\right\|\|x\| \|\right.\right.\right.
$$

for all $x \in \operatorname{span}\left\{e_{1}, \ldots, e_{n}\right\}$. Define a seminorm $\rho$ on $E$ by

$$
\rho(x)=\sup _{n}\left(1+\eta_{n}\right) \max _{1 \leq j \leq n} \max _{w \in W_{j}}\left|\left\langle P_{j} x, w\right\rangle\right| .
$$

We will show that $\rho$ is an equivalent norm on $E$, and the set

$$
W=\left\{\left(1+\eta_{n}\right) P_{j}^{\prime} w: n \in \mathbb{N}, 1 \leq j \leq n, w \in W_{j}\right\}
$$


is a countable p.n. subset of $(E, \rho)^{\prime}$. Then $E$ is isomorphically polyhedral by Fonf's Theorem (Theorem 2). Now let $x \in E$. By (b), there exists $m$ such that $\|x\| \|=$ $\left\|\left|P_{m} x \|\right|\right.$. Hence, by (3), and the fact that $\left(e_{n}\right)$ is monotone with respect to $\|\|\cdot\|$,

$$
\begin{aligned}
\|x\| \| & =\left\|\left|P_{m} x \| \leq\left(1+\epsilon_{m}\right) \max _{w \in W_{m}}\right|\left\langle P_{m} x, w\right\rangle \mid\right. \\
& \leq\left(1+\eta_{m}\right) \max _{w \in W_{m}}\left|\left\langle P_{m} x, w\right\rangle\right| \\
& \leq \rho(x) \leq 2\||| x \mid\| .
\end{aligned}
$$

Thus $\rho$ is an equivalent norm on $E$. Next we show that the supremum in (4) is attained. Fix $x \in E$. Choose sequences $n_{1} \leq n_{2} \leq \cdots,\left(j_{k}\right)$, and $\left(w_{k}\right)$ such that $1 \leq j_{k} \leq n_{k}, w_{k} \in W_{n_{k}}$ for all $k$, and $\rho(x)=\lim _{k}\left(1+\eta_{n_{k}}\right)\left|\left\langle P_{j_{k}} x, w_{k}\right\rangle\right|$. First assume

that $\lim _{k} n_{k}=\infty$. Then $\eta_{n_{k}} \rightarrow 0$. Since $\left(e_{n}\right)$ is monotone with respect to \|\|$\cdot\|\|$, we have $\rho(x) \leq\|\| x\|\|$. But there exists $k$ such that $\left\|\left|x\|\|=\left\|\mid P_{k} x\right\| \|\right.\right.$, and there is a $w \in W_{k}$ such that $\left\|\left|P_{k} x\right|\right\| \leq\left(1+\epsilon_{k}\right)\left|w\left(P_{k} x\right)\right|$. Thus

$$
\rho(x) \geq\left(1+\eta_{k}\right)\left|w\left(P_{k} x\right)\right| \geq \frac{1+\eta_{k}}{1+\epsilon_{k}}|\|x|\|\mid>\| x\|\|,
$$

a contradiction. Therefore, $\lim _{k} n_{k} \neq \infty$. By going to a subsequence, we may assume that $\left(n_{k}\right)$ is bounded. Using a further subequence if necessary, we may even assume it is constant. Thus the supremum in (A) is attained. From this it readily follows that the set $W$ is a p.n. subset of $(E, \rho)^{\prime}$. The countability of $W$ is evident.

Remark The assumption that the basis $\left(e_{n}\right)$ is shrinking is used only in the proof of $(\mathrm{a}) \Rightarrow(\mathrm{b})$. If $\left(e_{n}\right)$ is assumed to be unconditional and (a) holds, then $\left(e_{n}\right)$ must be shrinking. For otherwise $E$ contains a copy of $\ell^{1}$, which contradicts (a) by Fonf's Theorem (Theorem 1). Thus the assumption of shrinking is not needed if $\left(e_{n}\right)$ is unconditional.

\section{Orlicz sequence spaces}

In this section, we apply Theorem 3 to identify a class of isomorphically polyhedral Orlicz sequence spaces. Terms and notation about Orlicz sequence spaces follow that of [3]. An Orlicz function $M$ is a continuous non-decreasing convex function defined for $t \geq 0$ such that $M(0)=0$ and $\lim _{t \rightarrow \infty} M(t)=\infty$. If $M(t)>0$ for all $t>0$, then it is 
non-degenerate. Clearly a non-degenerate Orlicz function must be strictly increasing. The Orlicz sequence space $\ell_{M}$ associated with an Orlicz function $M$ is the space of all sequences $\left(a_{n}\right)$ such that $\sum M\left(\left|a_{n}\right| / \rho\right)<\infty$ for some $\rho>0$, equipped with the norm

$$
\|x\|=\inf \left\{\rho>0: \sum M\left(\left|a_{n}\right| / \rho\right)<\infty\right\} .
$$

Let $e_{n}$ denote the vector whose sole nonzero coordinate is a 1 at the $n$-th position. Then clearly $\left(e_{n}\right)$ is a basic sequence in $\ell_{M}$. The closed linear span of $\left\{e_{n}\right\}$ in $\ell_{M}$ is denoted by $h_{M}$. Alternatively, $h_{M}$ may be described as the set of all sequences $\left(a_{n}\right)$ such that $\sum M\left(\left|a_{n}\right| / \rho\right)<\infty$ for every $\rho>0$. Additional results and references on Orlicz spaces may be found in [3]. For a real null sequence $\left(a_{n}\right)$, let $\left(a_{n}^{*}\right)$ denote the decreasing rearrangement of the sequence $\left(\left|a_{n}\right|\right)$.

Theorem 4 Let $M$ be a non-degenerate Orlicz function such that there exists a finite number $K$ satisfying $\lim _{t \rightarrow 0} M(K t) / M(t)=\infty$. Then $h_{M}$ is isomorphically polyhedral.

Proof: For all $k \in \mathbb{N}$, let

$$
b_{k}=\inf \left\{\frac{M(K t)}{M(t)}: 0<t \leq M^{-1}\left(\frac{1}{k}\right)\right\} \text {. }
$$

Then $\lim _{k \rightarrow \infty} b_{k}=\infty$. Thus there is a sequence $\left(\eta_{k}\right)$ decreasing to 1 such that $\eta_{k}>$ $\left(1-b_{k+1}^{-1}\right)^{-1}$ for all $k$. Define a seminorm on $h_{M}$ by

$$
\|\|\left(a_{n}\right)\|\|=\sup _{k} \eta_{k}\left\|\left(a_{1}^{*}, \ldots, a_{k}^{*}, 0, \ldots\right)\right\|
$$

where $\|\cdot\|$ is the given norm on $h_{M}$. It is clear that \|\|$\cdot \| \mid$ is an equivalent norm on $h_{M}$, and that $\left(e_{n}\right)$ is a monotone basis with respect to \|\|$\cdot\|\|$. It suffices to show that $\||\| \cdot| \mid$ satisfy the remaining condition in part (b) of Theorem 3. We first show that if $\left(a_{n}\right)$ is a positive decreasing sequence in $h_{M}$, then there is a $k$ such that

$$
\left\|\left(a_{n}\right)\right\| \leq \eta_{k}\left\|\left(a_{1}, \ldots, a_{k}, 0, \ldots\right)\right\| .
$$

Assume otherwise. There is no loss of generality in assuming that $\left\|\left(a_{n}\right)\right\|=1$. Then $\sum M\left(a_{n}\right)=1$ and $\sum_{n=1}^{k} M\left(\eta_{k} a_{n}\right) \leq 1$ for all $k$. In particular, note that the second condition implies $a_{k} \leq M^{-1}(1 / k)$ for all $k$, since $\eta_{k} \geq 1$ and $\left(a_{n}\right)$ is decreasing. Now choose $m$ such that $\left\|\left(0, \ldots, 0, a_{m}, a_{m+1}, \ldots\right)\right\| \leq K^{-1}$. Then $\sum_{n=m}^{\infty} M\left(K a_{n}\right) \leq 1$. Also 
$M\left(K a_{n}\right) \geq b_{m} M\left(a_{n}\right)$ for all $n \geq m$. Therefore,

$$
\begin{aligned}
1 & =\sum M\left(a_{n}\right)=\sum_{n=1}^{m-1} M\left(a_{n}\right)+\sum_{n=m}^{\infty} M\left(a_{n}\right) \\
& \leq \eta_{m-1}^{-1} \sum_{n=1}^{m-1} M\left(\eta_{m-1} a_{n}\right)+b_{m}^{-1} \sum_{n=m}^{\infty} M\left(K a_{n}\right) \\
& \leq \eta_{m-1}^{-1}+b_{m}^{-1}<1,
\end{aligned}
$$

a contradiction. Hence (7) holds for some $k$. Now for a general element $\left(a_{n}\right) \in h_{M}$, choose $m$ such that $\left\|\left(a_{n}\right)\right\|=\left\|\left(a_{n}^{*}\right)\right\| \leq \eta_{m}\left\|\left(a_{1}^{*}, \ldots, a_{m}^{*}, 0, \ldots\right)\right\|$. Note that since $\lim _{k} \eta_{k}\left\|\left(a_{1}^{*}, \ldots, a_{k}^{*}, 0, \ldots\right)\right\|=\left\|\left(a_{n}\right)\right\|$, the supremum in equation (6) is attained, say, at $j$. Then choose $i$ large enough that $a_{1}^{*}, \ldots, a_{j}^{*}$ are found in $\left\{\left|a_{1}\right|, \ldots,\left|a_{i}\right|\right\}$. With this choice of $i$,

$$
\left\|\left(a_{1}, \ldots, a_{i}, 0, \ldots\right)\right\|\left|\geq \eta_{j}\left\|\left(a_{1}^{*}, \ldots, a_{j}^{*}, 0, \ldots\right)\right\|=\left\|\left|\left(a_{n}\right)\right|\right\|\right.
$$

by choice of $j$. Since the reverse inequality is obvious,

$$
\left\|\left(a_{n}\right)\right\|\left|=\left\|\left(a_{1}, \ldots, a_{i}, 0, \ldots\right) \mid\right\|,\right.
$$

as required.

\section{A counterexample}

Theorem 5 Let $M$ be a non-degenerate Orlicz function. Suppose there exists a sequence $\left(t_{n}\right)$ decreasing to 0 such that

$$
\sup _{n} \frac{M\left(K t_{n}\right)}{M\left(t_{n}\right)}<\infty
$$

for all $K<\infty$. Then $h_{M}$ is not isomorphically polyhedral.

Proof: Suppose that $h_{M}$ is isomorphically polyhedral . By Theorem 3 and the remark following it, one obtains a norm \|\|$\cdot \| \mid$ on $h_{M}$ as prescribed by part (b) of the theorem. Fix $\alpha>0$ so that $\left\|\left|x\|\mid \leq \alpha \Rightarrow\| x \| \leq 1\right.\right.$. Choose a sequence $\left(\eta_{k}\right)$ strictly decreasing to 
1. Let $n_{1}=\min \left\{n \in \mathbb{N}: \eta_{1}||\left|t_{n} e_{1}\right| \| \leq \alpha\right\}$. If $n_{1} \leq n_{2} \leq \cdots \leq n_{k}$ are chosen so that $\eta_{k}||\left|\sum_{j=1}^{k} t_{n_{j}} e_{j}\right||| \leq \alpha$, then $\eta_{k+1}||\left|\sum_{j=1}^{k} t_{n_{j}} e_{j}\right| \|<\alpha$. Hence

$$
\left\{n \geq n_{k}: \eta_{k+1}||\left|\sum_{j=1}^{k} t_{n_{j}} e_{j}+t_{n} e_{k+1}\right| \| \leq \alpha\right\} \neq \emptyset .
$$

Now define

$$
n_{k+1}=\min \left\{n \geq n_{k}: \eta_{k+1}||\left|\sum_{j=1}^{k} t_{n_{j}} e_{j}+t_{n} e_{k+1}\right||| \leq \alpha\right\} .
$$

This inductively defines a (not necessarily strictly) increasing sequence $\left(n_{k}\right)$ satisfying

$$
\eta_{k}||\left|\sum_{j=1}^{k} t_{n_{j}} e_{j}\right| \| \leq \alpha
$$

for all $k$ and the minimality condition (8). In particular, ||$\sum_{j=1}^{k} t_{n_{j}} e_{j} \mid \| \leq \alpha$ for all $k$, so $\left\|\sum_{j=1}^{k} t_{n_{j}} e_{j}\right\| \leq 1$ by the choice of $\alpha$. Therefore $\sum_{j=1}^{k} M\left(t_{n_{j}}\right) \leq 1$ for all $k$. For all $K<\infty$ and all $k \in \mathbb{N}$,

$$
\sum_{j=1}^{k} M\left(K t_{n_{j}}\right) \leq \sup _{m} \frac{M\left(K t_{m}\right)}{M\left(t_{m}\right)} \sum_{j=1}^{k} M\left(t_{n_{j}}\right) \leq \sup _{m} \frac{M\left(K t_{m}\right)}{M\left(t_{m}\right)} .
$$

Consequently, $\sum_{j=1}^{\infty} M\left(K t_{n_{j}}\right)<\infty$ for all $K<\infty$. Hence $x=\sum_{j=1}^{\infty} t_{n_{j}} e_{j}$ converges in $h_{M}$. Clearly $\||x|\|=\lim _{k} \mid\left\|\sum_{j=1}^{k} t_{n_{j}} e_{j}\right\| \leq \alpha$. We claim that in fact $\||x|\|=\alpha$. Otherwise, suppose $\|\mid x\| \|=\beta<\alpha$. Since $\left(e_{n}\right)$ is monotone with respect to $\|\cdot\| \|$, $\left\|\left|\sum_{j=1}^{k} t_{n_{j}} e_{j} \|\right| \leq \beta<\alpha\right.$ for all $k$. By the convergence of $x, \lim _{j} t_{n_{j}}=0$. So one can find $i$ such that $\left\|\mid t_{n_{i}} e_{j}\right\| \leq \leq \alpha-\beta$ for all $j$. Then

$$
\left\||| \sum_{j=1}^{i} t_{n_{j}} e_{j}+t_{n_{i}} e_{i+1}|\| \leq||| \sum_{j=1}^{i} t_{n_{j}} e_{j}|||+||| t_{n_{i}} e_{i+1}|| \mid \leq \beta+\alpha-\beta=\alpha .\right.
$$

By the minimality condition (8), $n_{i+1}=n_{i}$. Similarly, we see that $n_{j}=n_{i}$ for all $j \geq i$. This contradicts the convergence of $x$ and proves the claim. But now, by (9), $\left\||| \sum_{j=1}^{k} t_{n_{j}} e_{j}|\|<\alpha=|\| x|||\right.$ for all $k$, contradicting the choice of the norm $|\||\cdot|\|$.

We now construct an Orlicz function $M$ satisfying Theorem 5 while $h_{M}$ is $c_{0^{-}}$ saturated. We begin with some simple results which help to identify the $c_{0}$-saturated Orlicz sequence spaces. 
Proposition 6 Let $M$ be a non-degenerate Orlicz function. Then the following are equivalent.

(a) $h_{M}$ is $c_{0}$-saturated;

(b) $h_{M}$ does not contain an isomorph of $\ell^{p}$ for any $1 \leq p<\infty$;

(c) for all $q<\infty$,

$$
\sup _{0<\lambda, t \leq 1} \frac{M(\lambda t)}{M(\lambda) t^{q}}<\infty .
$$

Proof: Clearly (a) implies (b). If (a) fails, let $Y$ be an infinite dimensional closed subspace of $h_{M}$ which contains no isomorph of $c_{0}$. By [3, Proposition 4.a.7], $Y$ has a subspace $Z$ isomorphic to some Orlicz sequence space $h_{N}$. Then $h_{N}$ contains no isomorph of $c_{0}$. By [3, Theorem 4.a.9], $h_{N}$ contains an isomorph of some $\ell^{p}, 1 \leq p<\infty$. Hence $Y$ contains a copy of $\ell^{p}$, and (b) fails. The equivalence of (b) and (c) also follows from [3, Theorem 4.a.9].

Proposition 7 Let $\left(b_{n}\right)_{n=0}^{\infty}$ be a decreasing sequence of strictly positive numbers such that

$$
\sup _{m, n} \frac{b_{m+n}}{b_{n}} K^{m}<\infty \text { for all } K<\infty .
$$

Define $M$ to be the continuous, piecewise linear function such that $M(0)=0$,

$$
M^{\prime}(t)=\left\{\begin{array}{lll}
b_{n} & \text { if } 2^{-n-1}<t<2^{-n}, n>0 \\
b_{0} & \text { if } 2^{-1}<t
\end{array}\right.
$$

Then $M$ is a non-degenerate Orlicz function so that $h_{M}$ is $c_{0}$-saturated.

Proof: It is clear that $M$ is a non-degenerate Orlicz function. For all $n \geq 0,2^{-n-1} b_{n} \leq$ $M\left(2^{-n}\right) \leq 2^{-n} b_{n}$. Hence

$$
C_{q} \equiv \sup _{m, n} \frac{M\left(2^{-m-n}\right)}{M\left(2^{-n}\right)} 2^{m q} \leq 2 \sup _{m, n} \frac{b_{m+n}}{b_{n}}\left(2^{q-1}\right)^{m}<\infty
$$

for any $q<\infty$. Now if $\lambda, t \in(0,1]$, choose $m, n \geq 1$ such that $t \in\left(2^{-m}, 2^{-m+1}\right.$ ], $\lambda \in\left(2^{-n}, 2^{-n+1}\right]$. Then $\lambda t \in\left(2^{-m-n}, 2^{-m-n+2}\right]$. If $m \geq 2$, then

$$
\frac{M(\lambda t)}{M(\lambda) t^{q}} \leq 2^{2 q} \frac{M\left(2^{-(m-2)-n}\right)}{M\left(2^{-n}\right)} 2^{(m-2) q} \leq 4^{q} C_{q} .
$$


If $m=1$, then $t>2^{-1}$. Therefore

$$
\frac{M(\lambda t)}{M(\lambda) t^{q}} \leq t^{-q} \leq 2^{q}
$$

Thus

$$
\sup _{0<\lambda, t \leq 1} \frac{M(\lambda t)}{M(\lambda) t^{q}}<\infty,
$$

and $h_{M}$ is $c_{0}$-saturated by the previous proposition.

Theorem 8 There exists an Orlicz function $M$ such that $h_{M}$ is $c_{0}$-saturated but not isomorphically polyhedral. In particular, a $c_{0}$-saturated space with a separable dual is not necessarily isomorphically polyhedral.

Proof: It is well known that every $c_{0}$-saturated space $h_{M}$ has a separable dual. Thus the second statement follows from the first. Let $\alpha_{0}=\alpha_{1}=\alpha_{2}=1$, and let $\alpha_{j}=(e / j)^{j}$ for $j \geq 3$. Then $\left(\alpha_{j}\right)$ is a decreasing sequence. Choose a decreasing sequence $\left(c_{j}\right)_{j=0}^{\infty}$ of strictly positive numbers such that $c_{j+1} \leq \alpha_{j} \alpha_{2 j^{2}} c_{j}$ for all $j \geq 0$. For convenience, set $s_{n}=\sum_{j=1}^{n} j$ for all $n \geq 1$. Now define $b_{0}=c_{0}, b_{1}=c_{1}$, and $b_{s_{n}+k}=c_{n+1} / \alpha_{n+1-k}$ whenever $n \geq 1$ and $1 \leq k \leq n+1$. We first show that the sequence $\left(b_{j}\right)$ satisfies the conditions in Proposition 0 .

Claim $1\left(b_{j}\right)$ is a decreasing sequence.

One verifies directly that $b_{0} \geq b_{1} \geq b_{2}$. If $n \geq 1$ and $1 \leq k \leq j \leq n+1$,

$$
b_{s_{n}+k}=\frac{c_{n+1}}{\alpha_{n+1-k}} \geq \frac{c_{n+1}}{\alpha_{n+1-j}}=b_{s_{n}+j}
$$

since $\left(\alpha_{m}\right)$ is decreasing. Finally,

$$
b_{s_{n+1}+1}=\frac{c_{n+2}}{\alpha_{n+1}} \leq \alpha_{2(n+1)^{2}} c_{n+1} \leq c_{n+1}=b_{s_{n}+n+1}
$$

for all $n \geq 1$. This proves Claim 1 .

Claim $2 \quad b_{m+n} \leq \alpha_{m} b_{n}$ for all $m \geq 0, n \geq 2$.

Express $n=s_{i}+k, m+n=s_{j}+l$, where $1 \leq i \leq j, 1 \leq k \leq i+1$, and $1 \leq l \leq j+1$. If $i=j$, then $l-k=m$. Moreover, $i+1-k \geq \max \{l-k, i+1-l\}$, from which it follows that $\alpha_{i+1-k} \leq \alpha_{l-k} \alpha_{i+1-l}$. Therefore,

$$
b_{m+n}=\frac{c_{i+1}}{\alpha_{i+1-l}} \leq \alpha_{m} \frac{c_{i+1}}{\alpha_{i+1-k}}=\alpha_{m} b_{n} .
$$


Now consider the possibility that $j>i$. Note first that

$$
m=(m+n)-n \leq s_{j}+j+1-\left(s_{i}+1\right) \leq s_{j}+j \leq 2 j^{2} .
$$

Hence $\alpha_{m} \geq \alpha_{2 j^{2}}$. Using Claim 1 and the properties of the sequence $\left(c_{j}\right)$, we obtain

$$
\begin{aligned}
b_{m+n} & \leq b_{s_{j}+1}=\frac{c_{j+1}}{\alpha_{j}} \\
& \leq \alpha_{2 j^{2}} c_{j} \leq \alpha_{m} c_{i+1} \\
& =\alpha_{m} b_{s_{i}+i+1} \leq \alpha_{m} b_{n} .
\end{aligned}
$$

$\underline{\text { Claim } 3}$

$$
\sup _{m, n} \frac{b_{m+n}}{b_{n}} K^{m}<\infty \text { for all } K<\infty .
$$

First observe that for $i \geq 1,1 \leq k \leq i+1$, and $K<\infty$,

$$
\begin{aligned}
b_{s_{i}+k} K^{s_{i}+k} & =\frac{c_{i+1}}{\alpha_{i+1-k}} K^{s_{i}+k} \leq \alpha_{2 i^{2}} c_{i} K^{s_{i}+i+1} \\
& \leq c_{0} \alpha_{2 i^{2}} K^{s_{i}+i+1} \rightarrow 0
\end{aligned}
$$

as $i \rightarrow \infty$. Hence $\left(b_{m} K^{m}\right)_{m}$ is bounded. Therefore $\sup _{n=1,2} \sup _{m} b_{m+n} K^{m} / b_{n}<\infty$. On the other hand, using Claim 2,

$$
\sup _{n \geq 2} \sup _{m} \frac{b_{m+n}}{b_{n}} K^{m} \leq \sup _{m} \alpha_{m} K^{m}<\infty
$$

by direct verification.

Define the function $M$ using the sequence $\left(b_{j}\right)$ as in Proposition 7. Using Claims 1 and 3 , and the proposition, we see that $h_{M}$ is $c_{0}$-saturated. To complete the proof, it suffices to find a sequence $\left(t_{n}\right)$ as in Theorem 5. We claim that the sequence $\left(t_{n}\right)=$ $\left(2^{-s_{n}}\right)$ will do. Clearly $\left(t_{n}\right)$ decrease to 0 . Fix $m \in \mathbb{N}$. For all $n>m$,

$$
b_{s_{n}-m}=b_{s_{n-1}+(n-m)}=\frac{c_{n}}{\alpha_{m}} .
$$

Hence

$$
\begin{aligned}
M\left(2^{m} t_{n}\right) & =M\left(2^{-s_{n}+m}\right) \leq \frac{b_{s_{n}-m}}{2^{s_{n}-m}} \\
& =\frac{c_{n}}{\alpha_{m} 2^{s_{n}-m}}=\frac{2^{m+1}}{\alpha_{m}} \frac{c_{n}}{2^{s_{n}+1}} \\
& =\frac{2^{m+1}}{\alpha_{m}} \frac{b_{s_{n}}}{2^{s_{n}+1}} \leq \frac{2^{m+1}}{\alpha_{m}} M\left(t_{n}\right)
\end{aligned}
$$


whenever $n>m$. Therefore,

$$
\sup _{n} \frac{M\left(2^{m} t_{n}\right)}{M\left(t_{n}\right)}<\infty
$$

for all $m \in \mathbb{N}$.

The obvious question to be raised is how to characterize isomorphically polyhedral $h_{M}$ in terms of the Orlicz function $M$. We suspect that the condition given in Theorem 耳is the correct one. It can be shown that if $\liminf _{t \rightarrow 0} M(K t) / M(t)<\infty$ for all $K<\infty$, then for any sequence $\left(\eta_{k}\right)$ decreasing to 1 , the norm given by equation (6) does not satisfy part (b) of Theorem 3 .

\section{References}

[1] V. P. Fonf, On a property of Lindenstrauss-Phelps spaces, Funct. Anal. Appl. 13(1979), 79-80 (translated from Russian).

[2] V. P. Fonf, Polyhedral Banach spaces, Matematicheskie Zametki 30(1981), 627-634 (translated from Russian).

[3] Joram Lindenstrauss and Lior Tzafriri, "Classical Banach Spaces I, Sequence Spaces", Springer-Verlag, Berlin, 1977.

[4] H. Rosenthal, Class notes, Topics course in analysis, University of Texas at Austin.

[5] H. Rosenthal, Some aspects of the subspace structure of infinite dimensional Banach spaces, Approximation Theory and Functional Analysis (ed. C. Chuy), Academic Press, 1990.

Department of Mathematics

National University of Singapore

Singapore 0511

e-mail(bitnet) : matlhh@nusvm 